\title{
Effectiveness of Mind-Fullness Based Cognitive Therapy on Emotional Cognitive Regulation, Resiliency and Competitive Anxiety in Female Athletes
}

\author{
Sharzad Rockey ${ }^{1}$, Farah Naderi ${ }^{2, *}$, (D)
}

\begin{abstract}
${ }^{1}$ M.A., Department of Psychology, Faculty of Humanities, Ahvaz Branch, Islamic Azad University, Ahvaz, Iran ${ }^{2}$ Associate Professor, Department of Psychology, Faculty of Humanities, Ahvaz Branch, Islamic Azad University, Ahvaz, Iran

* Corresponding author: Farah Naderi, Associate Professor, Department of Psychology, Faculty of Humanities, Ahvaz Branch, Islamic Azad University, Ahvaz, Iran. E-mail: nmafrah@yahoo.com
\end{abstract}

Received: 28 Mar 2018

Accepted: 06 Sep 2018

Abstract
Introduction: Mindfulness is a skill that allows people to take incidents at a disadvantage in the present. The
aim of this study was to determine the effectiveness of mind-fullness based cognitive therapy on emotional
cognitive regulation, resiliency and competitive anxiety in female athletes.
Methods: This experimental study was a pre-test and post-test type with control group. The statistical
population were all women athletes in Ahwaz who were studied in 2017 academic year. The sample consisted
of 30 individuals who were selected by simple random sampling ( 15 in the experimental group and 15 in the
control group). Before and after intervention and one month later in follow-up process, the subjects
responded to research scales including resiliency, competitive anxiety and cognitive emotion regulation
questionnaires. The experimental group received cognitive therapy based on mind-awareness in 8 sessions
of 90 minutes each. But there was no intervention on the control group. To analyze the data, Multivariate
Covariance Analysis and One Way Covariance Analysis was applied.
Results: Findings showed that there was a significant difference between the experimental and control
groups in the cognitive-emotional regulation, resiliency and competitive anxiety (P < 0.0001). The results
were sustained at the follow-up stage.
Conclusions: According to the results, cognitive therapy based on mind-awareness can be used to improve
cognitive-emotional regulation, resiliency and competitive anxiety of female athletes.
Keywords: Mindfulness based Cognitive Therapy, Emotional Cognitive Regulation, Resiliency,
Competitive Anxiety, Female Athletes

(C) 2018 Iranian Nursing Scientific Association (INA) 


\title{
اثربخشى شناخت درماذى مبتنى بر ذهن آكاهى بر تنظيم شناختى هيجان، انعطاف يذيرى كنشى و اضطراب رقابتى ورزشكاران زن
}

\author{
(iD) شهرزاد راكى '، فرح نادرى
}

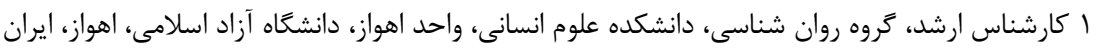

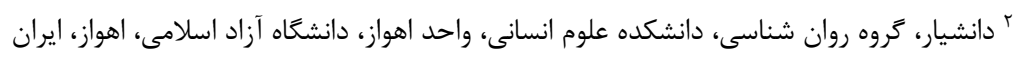
* نويسنده مسئول: فرح نادرى، دانشيار، كروه روان شناسى، دانشكده علوم انسانى، واحد اهواز، دانشعاه آزاداد اسلامى، اهواز، ايران. ايميل: nmafrah@yahoo.com

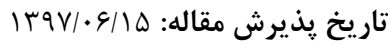

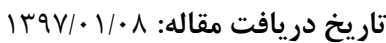

جكيده

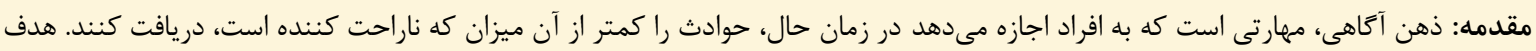

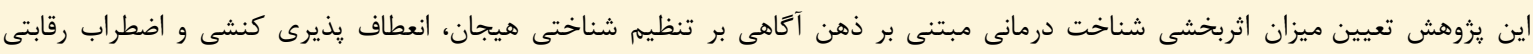
ورزشكاران زن بود.

روش كار: اين يزوهش تجربى از نوع پِيشآزمون و پِّآزمون با كروه كنترل بوده است. جامعه آمارى كليه زنان ورزشكار شهر اهواز بودند كه در سال

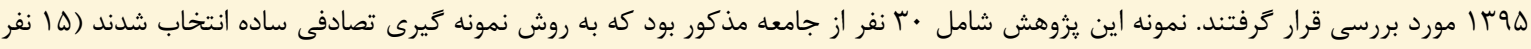

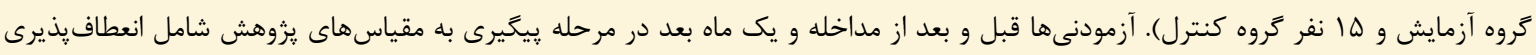

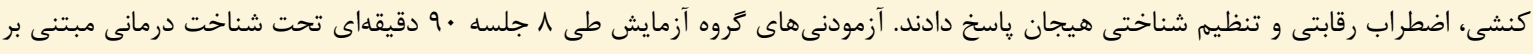

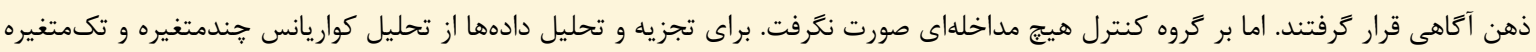
استفاده شد.

يافتهها: يافتهها نشان داد بين گروههاى آزمايش و كنترل در متغيرهاى تنظيم شناختى هيجان، انعطاف پذيرى كنشى و اضطراب رقابتى تفاوت . .

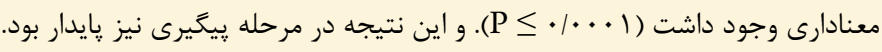

نتيجه كَيرى: با توجه به نتايج به دست آمده، مىتوان براى بهبود تنظيم شناختى هيجان، انعطاف پٍيرى كنشى و اضطراب رقابتى ورزشكاران زن از

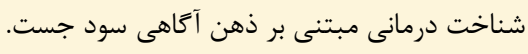
وازًَان كليدى: شناخت درمانى مبتنى بر ذهن آكاهى، تنظيم شناختى هيجان، انعطاف پِيرى كنشى، اضطراب رقابتى، ورزشكاران زن تمامى حقوق نشر براى انجمن علمى يرستارى ايران محفوظ است.

يافتن دست يافتن به آرامش، و اخذ نتايج بهترى از عمر خود استفاده

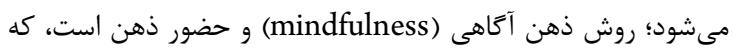

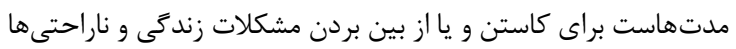

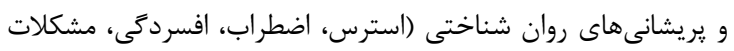

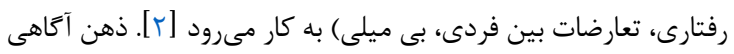

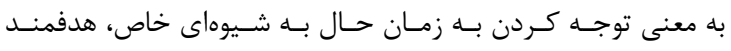

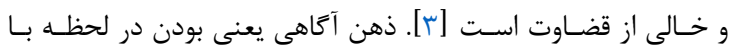

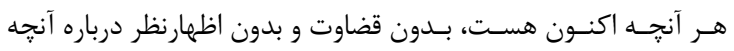

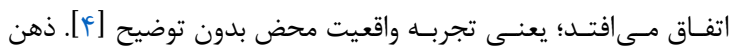

از آنجايى كه ورزشكاران براى رشد و كسب مدالهاى ورزشى مجبور به

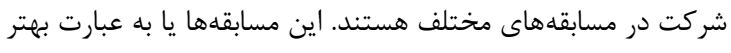

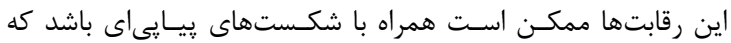

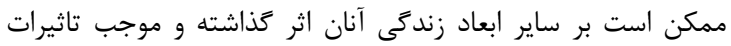

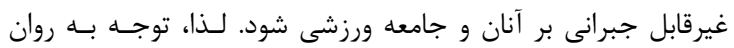

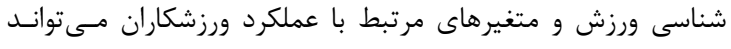

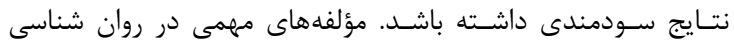

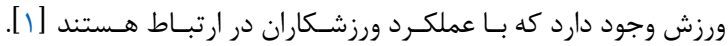

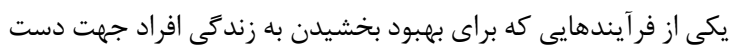


توانايى، يا يِيامد ساز كارى موفقيت آميز با شرايط تهديد كننده تعريف

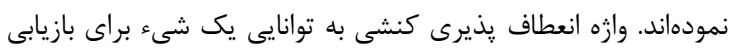

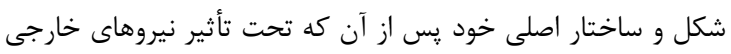

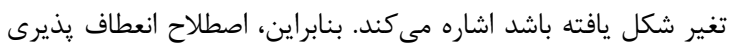

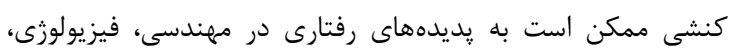

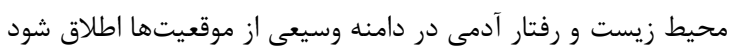

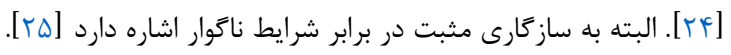

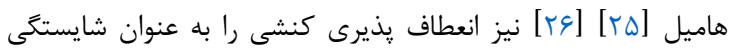

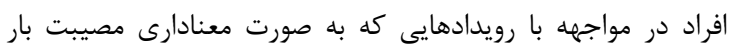

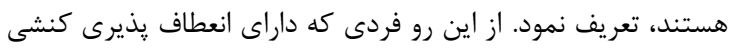

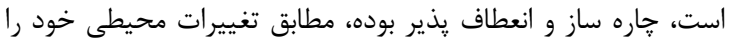

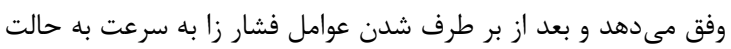

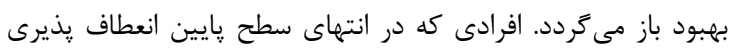

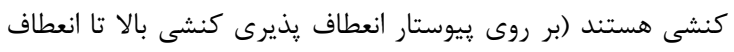

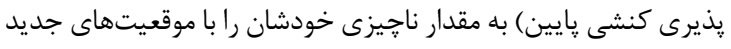

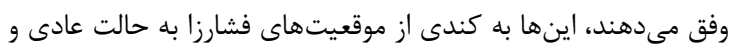

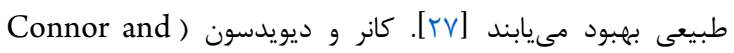

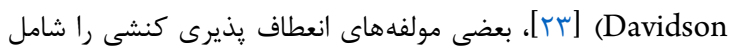

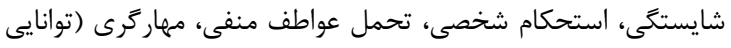

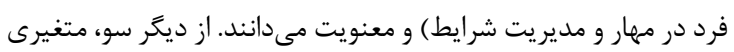

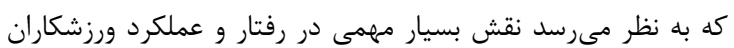

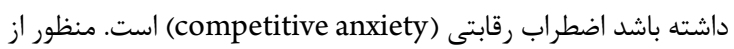

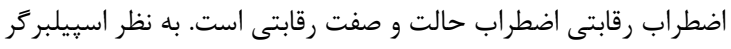

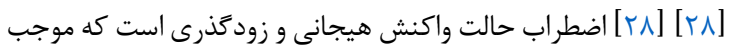

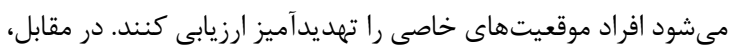

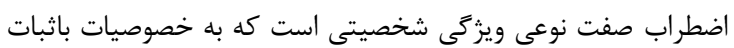

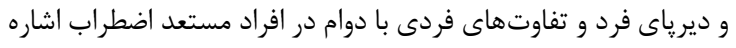

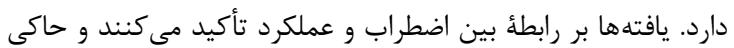

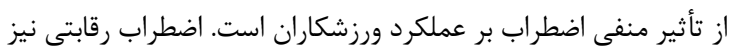

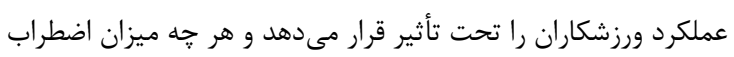

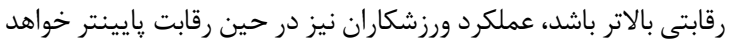

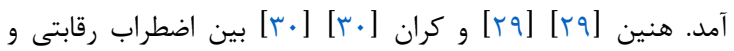

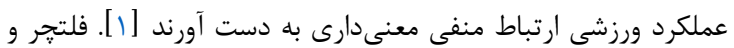

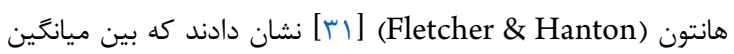

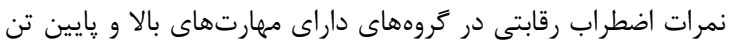

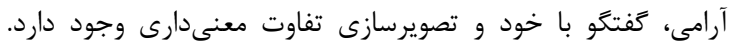

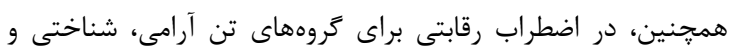

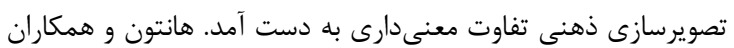
(Hanton et al) اضطراب جسمى ورزشكاران با نزديك شدن به مسابقه افزايش و اعتماد

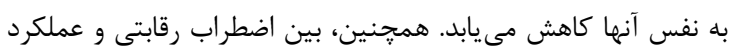

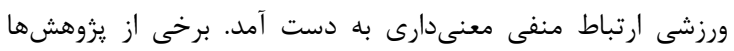

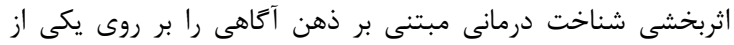

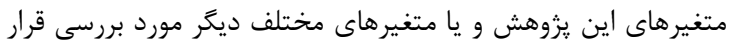

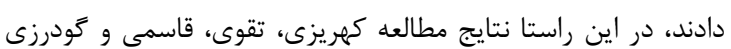

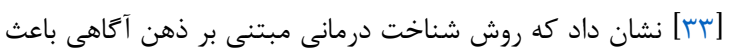

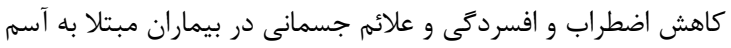

آكًاهى يك روش يا فن نيست، اكرجه در انجام آن روشهـا و فنون

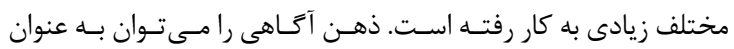

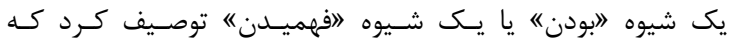

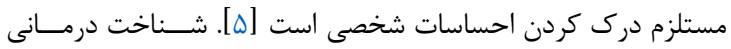

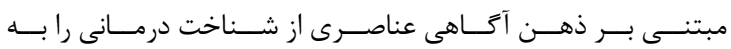

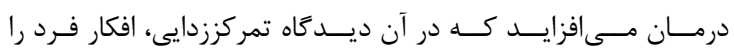

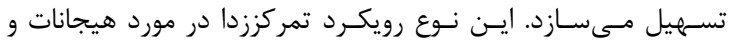

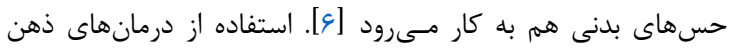

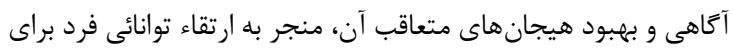

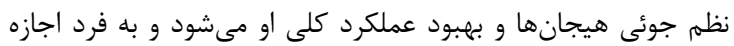

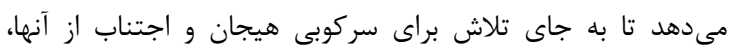

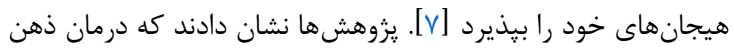

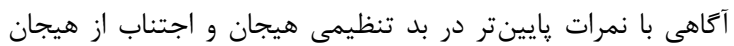

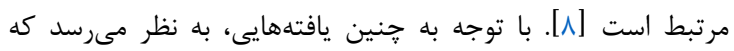

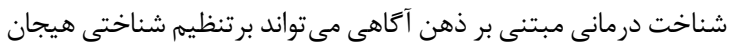

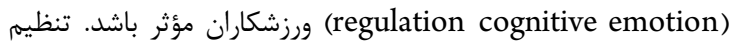

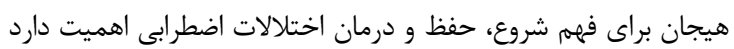

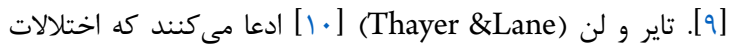

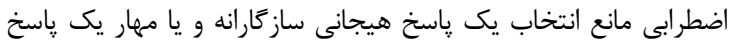

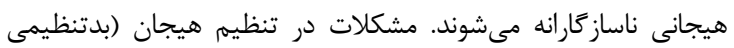

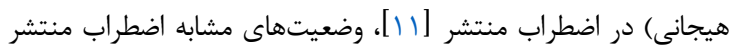

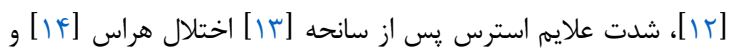

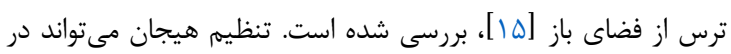

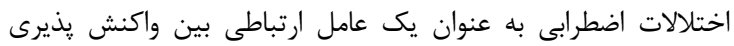

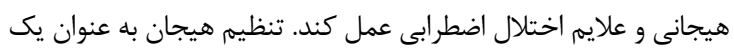

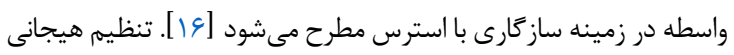

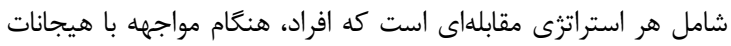

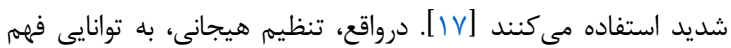

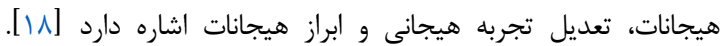

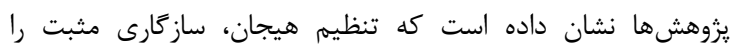

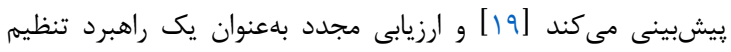

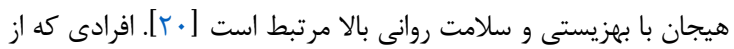

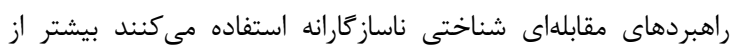

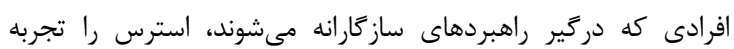

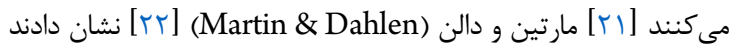

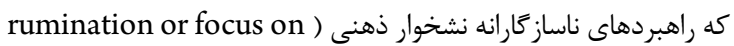

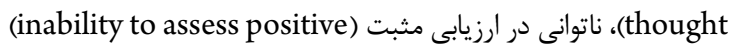
و سرزنش خود (self-blame)، پيش بيش (ينى كننده استرس هستند.

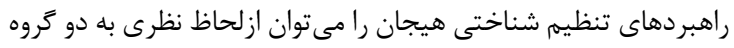

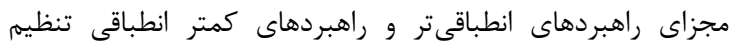

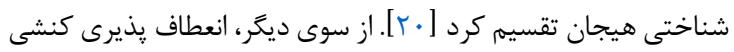

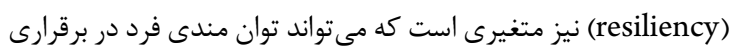

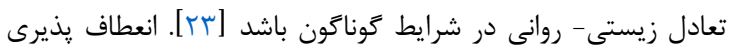

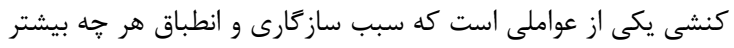

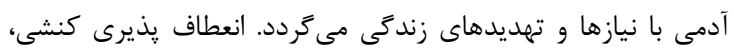

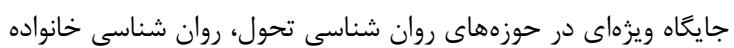

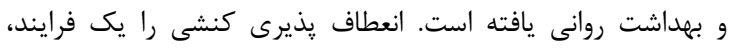


مهارتهاى ذهن آكاهى كاباتزين، استها، گولد آستين و سنتورلى دالى

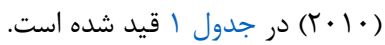

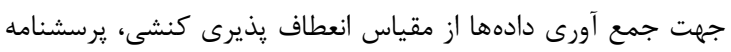
اضطراب رقابتى و مقياس تنظيم شناختى هيجان استفاده كرديد.

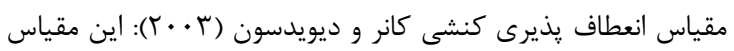

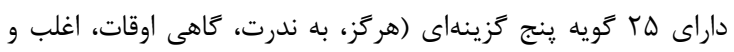

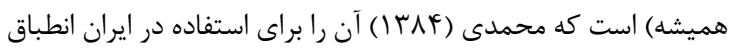

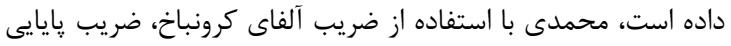

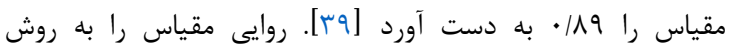

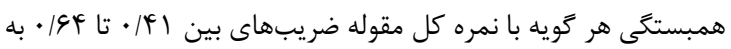

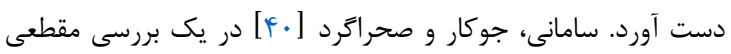

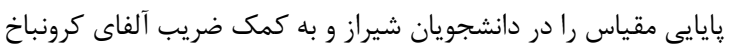

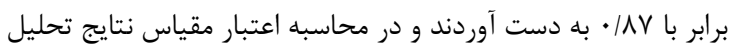

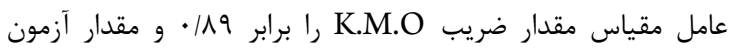

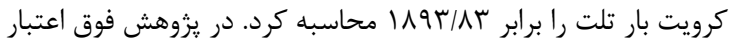

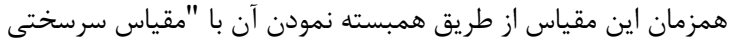

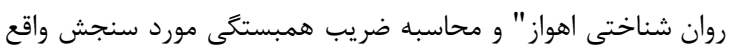

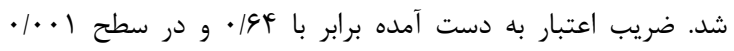

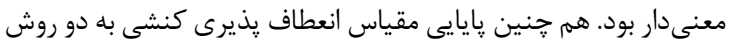

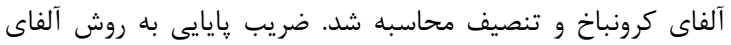

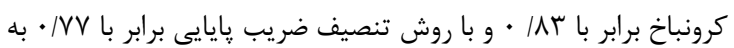

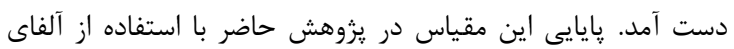

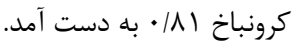
يرسشنامؤ اضطراب رقابتى: يرسشنامئ اضطراب رقابتى توسط مارتنز،

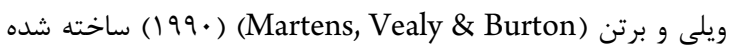

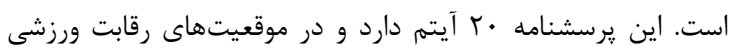

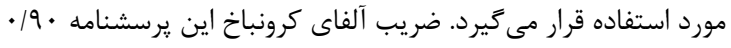

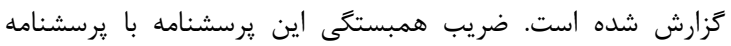

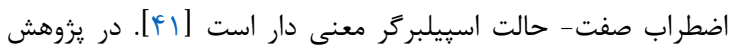

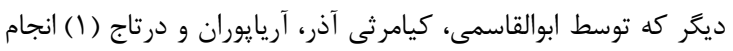

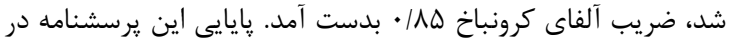

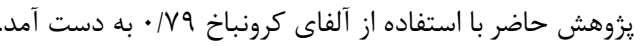

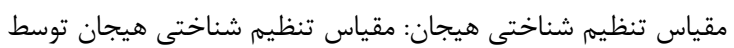

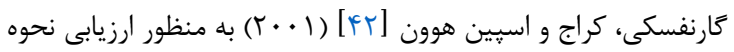

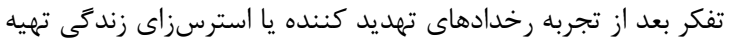

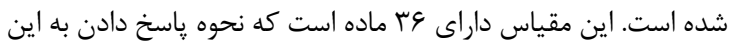

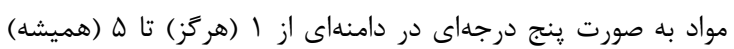

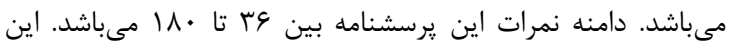

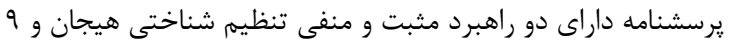

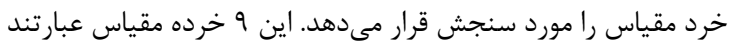

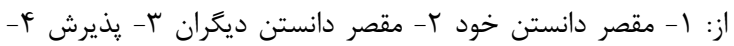

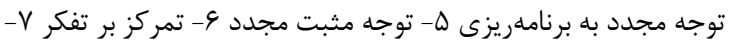

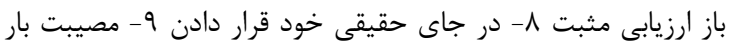

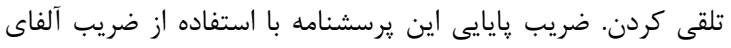

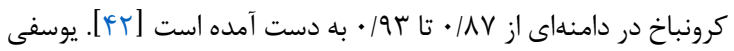

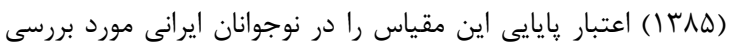

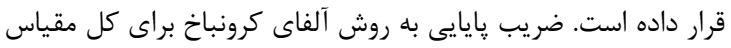

شده است. همجنين مطالعه كرد و رهبرى [ب [ب] نشان داد كه به

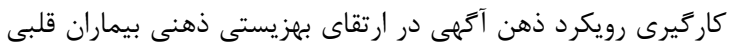

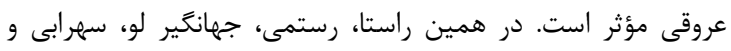

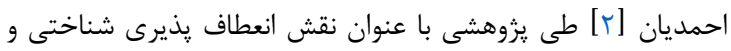

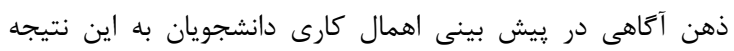

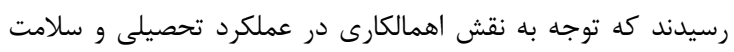

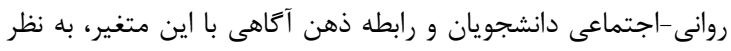

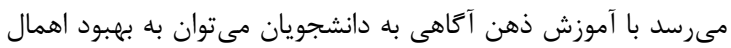

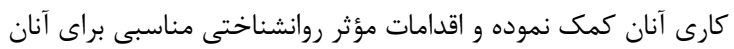

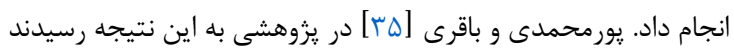

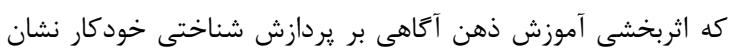

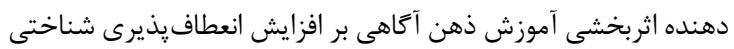

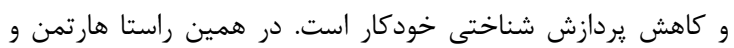

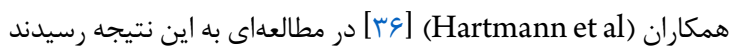
كه ذهن آكاهى مبتنى بر كاهش استرس، نتايج مثبت درمـانى و

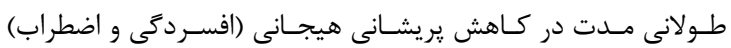

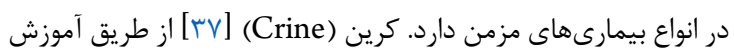

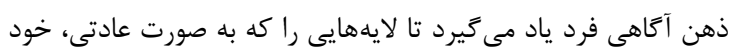

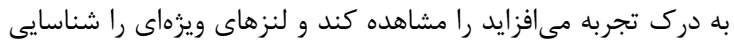

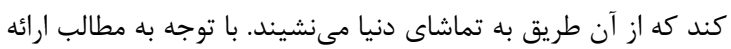

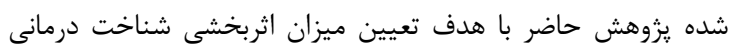

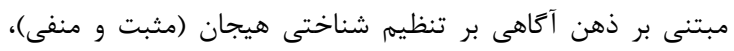

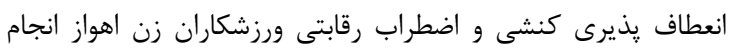

\section{روش كار}

در اين يزوهش از روش تجربى از نوع طرح بِيش آزمون و پِّ آزمون با

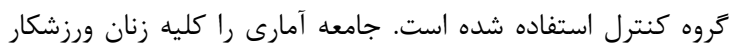

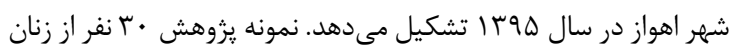

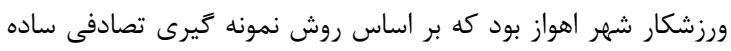

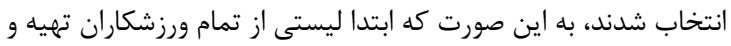

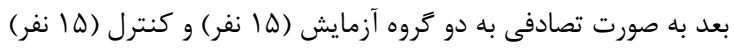

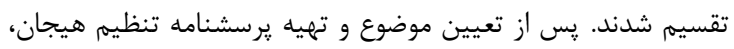

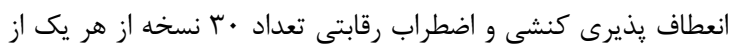

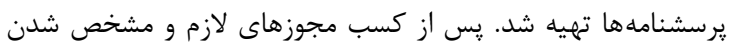

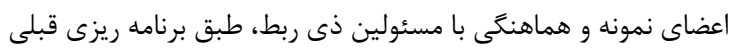

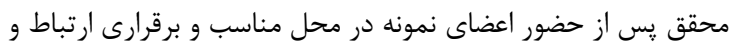

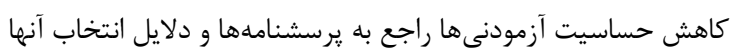

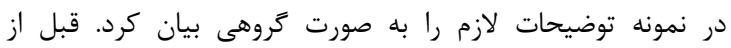

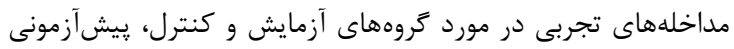

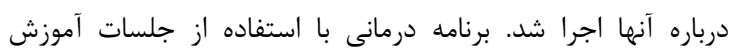

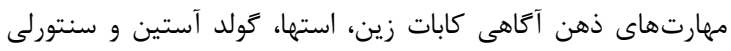
(Kabat-zinn, Stahl, Goldstein, santorelli)

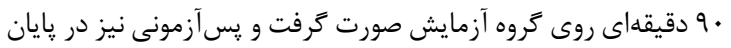

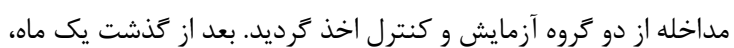

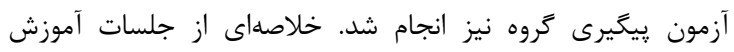


هايايى مناسب و رضايت بخشى براى استفاده در فرهنگ ايرانى برخوردار

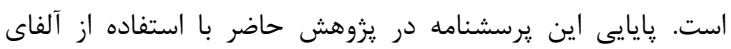

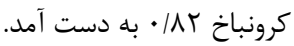

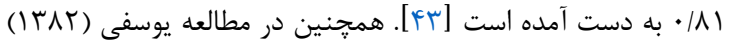

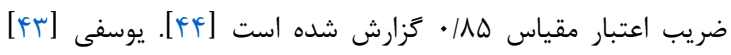

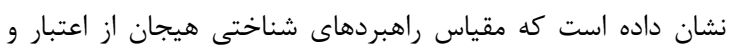

جدول ا: خلاصه جلسات آموزشى آموزش مهارتهاى ذهن آكاهى كابات زين، استها، كولد آستين و سنتورلى (• (†) جلسات

جلسه اول: مراقبه و تمرين آكاهى، تكنيك خوردن كشمش هأ دقيقه وارسى بدن و صحبت در مورد احساسات ناشى از آن و تكاليف خانگى حضور ذهن در لحظه و بست دادن تكنيك خوردن كشمش به ساير فعاليتها.

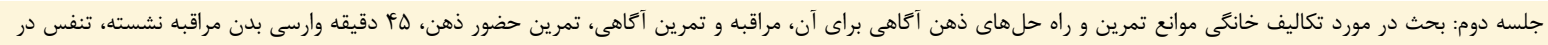

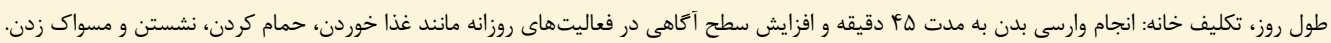

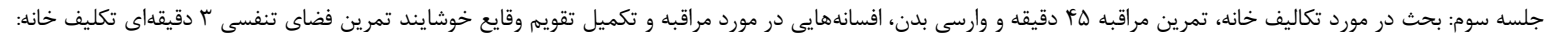

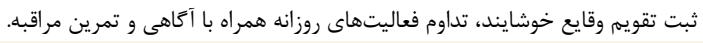

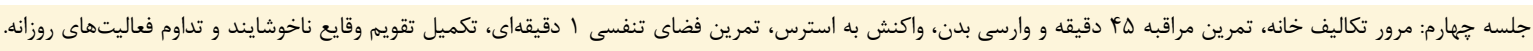

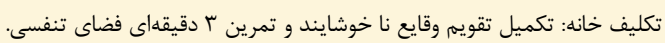

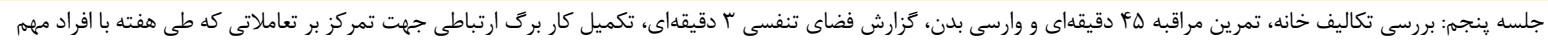

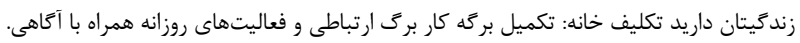

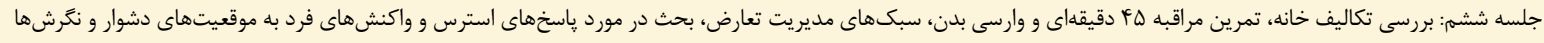

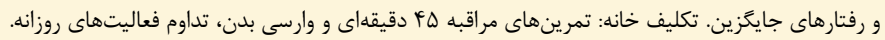

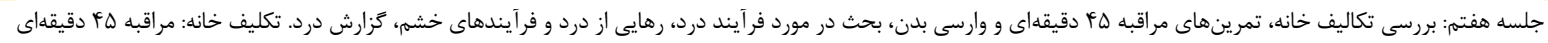

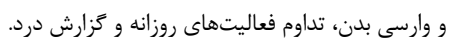

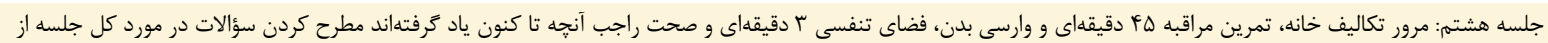

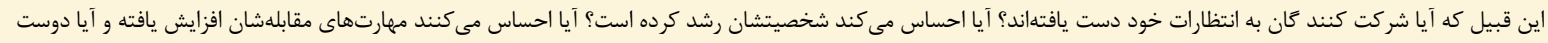
دارند مراقبه ادامه دهند.

فرض همًَنى ضرايب برقرار است. لذا در اين مرحله ابتدا آزمون تحليل

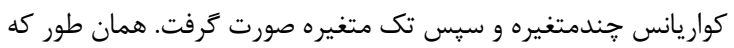

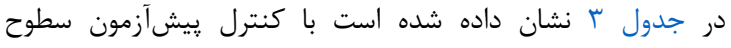

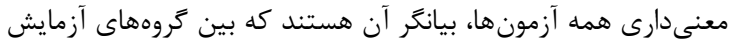

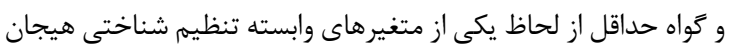

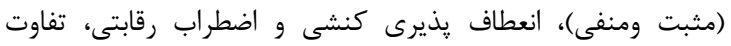

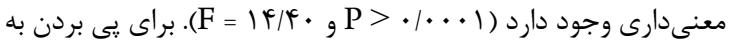

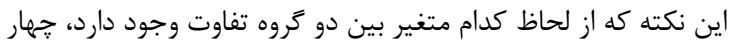

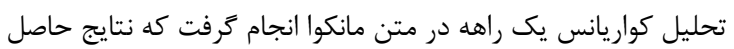

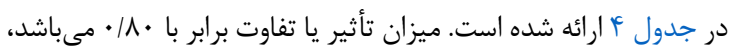

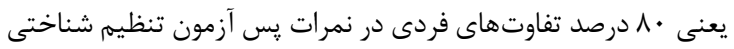

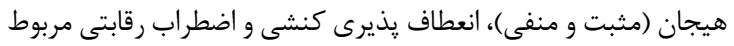

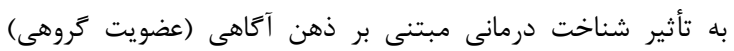

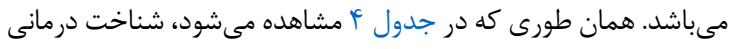

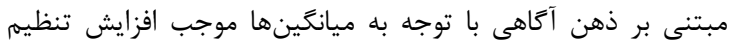

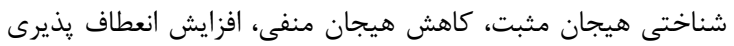

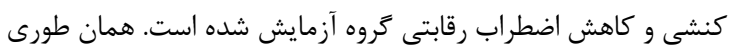

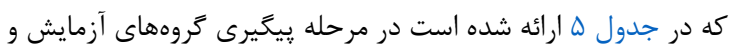

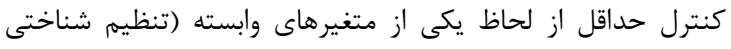

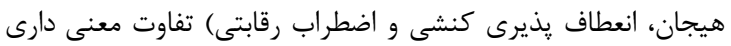

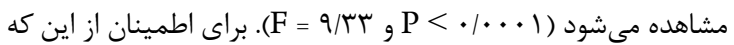

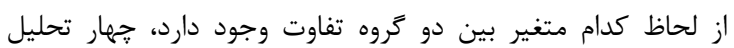

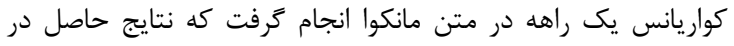

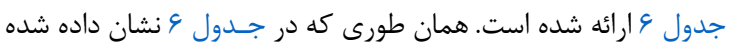

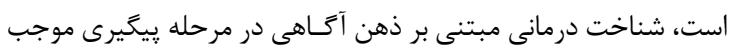

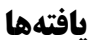

در تحقيق حاضر به طور كلى مىتوان كَفت گروهها از لحاظ متغيرهاى جمعيت شناختى تا حد زيادى همسان شدهاند. به طورى كورى كه ميانكَين

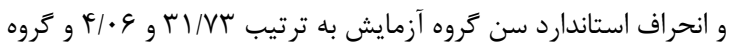

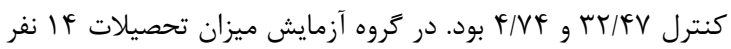

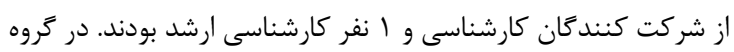

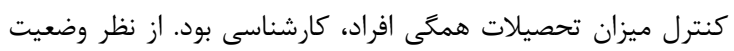

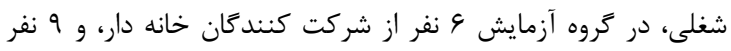

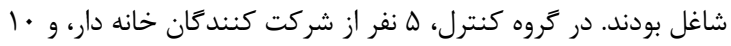

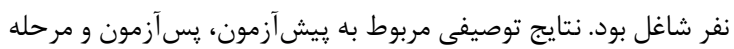

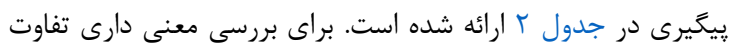

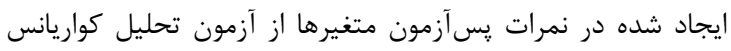

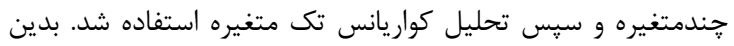

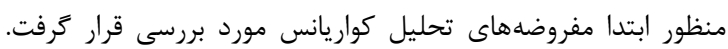
جهت بررسى مفروضه نرمال بودن توزيع متغيرها در كروهها از آز آزمون

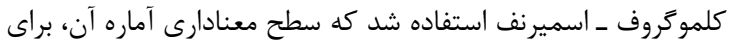

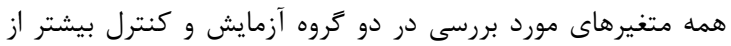

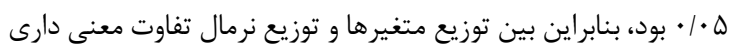
وجود نداشت. مفروضه همخنى واريانس متغيرهاى وابسته (نمرات

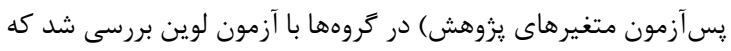

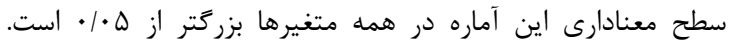

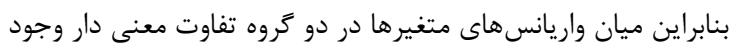

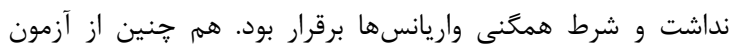

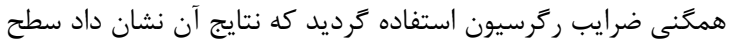

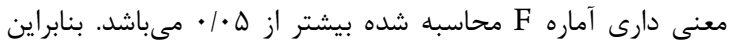
تفاوت معنى دارى ميان ضرايب ركرسيون مشاهده نغرديد و در نتيجه 
تداوم اثر تنظيم شناختى هيجان (مثبت ومنفى)، انعطاف يذيرى كنشى إنى إنى و اضطراب رقابتى كروه آزمايش شده است هيجان (مثنت

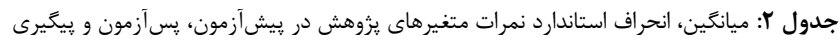

\begin{tabular}{|c|c|c|c|c|c|}
\hline \multicolumn{3}{|c|}{ تروه كنترل } & \multicolumn{3}{|c|}{ تروه آزمايش } \\
\hline بيگيرى & 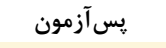 & بيش آزمون & يِيَيرى & يس آزمون & بيش آزمون \\
\hline \multicolumn{6}{|c|}{ انعطاف يذيرى كنشى } \\
\hline (انحراف معيار) & (انحراف معيار) & (انحر اف معيار) & (انحراف معيار) & (انحر اف معيار) & (انحراف معيار) \\
\hline$(\mathrm{A} / \mathrm{NT})$ & $(\Lambda / \Lambda \Delta)$ & $(q / \Gamma \Delta)$ & $(11 / r 9)$ & $(11 / \Gamma \Delta)$ & $(11 / \wedge r)$ \\
\hline ميانكَين & ميانكَين & ميانكَين & ميانگين & ميانغين & ميانغين \\
\hline$\Delta G / F Y$ & $\Delta \& / \Lambda$ & $\Delta 9 / 1$. & $\Delta g / T q$ & $\Delta \Delta / \wedge \Delta$ & $\mathrm{eV} / \mathrm{V}$. \\
\hline \multicolumn{6}{|c|}{ تنظيم شناختى هيجان مثبت } \\
\hline (انحراف معيار) & (انحراف معيار) & (انحراف معيار) & (انحراف معيار) & (انحراف معيار) & (انحر اف معيار) \\
\hline$(\varphi / \Gamma q)$ & $(F / \Delta \mid)$ & $(\boldsymbol{\Psi} / ৭ \Psi)$ & $(\uparrow / 19)$ & $(F / \Delta \mid)$ & $(\Delta / F V)$ \\
\hline ميانغين & ميانغين & ميانغين & ميانغين & ميانغين & ميانغين \\
\hline$T V / / 9$ & $T V / \cdot \Delta$ & $r V / \Delta \cdot$ & 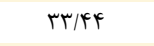 & $r \mu / \cdot \Delta$ & $r V / 9$. \\
\hline \multicolumn{6}{|c|}{ تنظيم شناختى هيجان منفى } \\
\hline (انحراف معيار) & (انحراف معيار) & (انحراف معيار) & (انحراف معيار) & (انحراف معيار) & (انحر اف معيار) \\
\hline$(r / \times q)$ & $(r / \Delta T)$ & $(\Gamma / 9 \Delta)$ & $(r / 99)$ & $(r / \Delta T)$ & $(\tau / \uparrow \Lambda)$ \\
\hline ميانغين & ميانكَين & ميانغَين & ميانكين & ميانغين & ميانگين \\
\hline$r V / r \Lambda$ & $r V / r$. & $r V / T \Delta$ & $r T / l V$ & $r r / 9$. & rब/r. \\
\hline \multicolumn{6}{|c|}{ اضطراب رقابتى } \\
\hline (انحراف معيار ) & (انحراف معيار) & (انحر اف معيار) & (انحراف معيار) & (انحر اف معيار) & (انحر اف معيار) \\
\hline$(\% / / 9)$ & $(T / F T)$ & $(r / \cdot v)$ & $(\Psi / \Delta r)$ & $(\kappa / \% \cdot)$ & (F/9T) \\
\hline ميانكَين & ميانكَين & ميانكَين & ميانغين & ميانكَين & ميانكَين \\
\hline$r V / 99$ & $r V / q \Delta$ & $r N / r$. & TF/VA & $r F / \Lambda$. & rN/A. \\
\hline
\end{tabular}

جدول با: نتايج تحليل كواريانس جندمتغيرى نمرات تنظيم شناختى هيجان (مثبت ومنفى)، انعطاف يذيرى كنشى و اضطراب رقابتى دركروهها

\begin{tabular}{|c|c|c|c|c|c|c|c|}
\hline توان آمارى & مجذور اتا & سطح معنى دارى & $\mathbf{F}$ & درجه آزادى خطا & درجه آزادى فرضيه & مقدار & نام آزمون \\
\hline $1 / \cdot$ & $\cdot / \Lambda$ & $\cdot 1 \cdot \cdots 1$ & $\mid f / f$. & rI & f & $\cdot / \Lambda$ & آزمون اثر پِيلايى \\
\hline $1 / \cdots$ & $\cdot 1 \Lambda$ & $\cdot 1 \cdots \cdot 1$ & $\mid f / 4$. & rI & r & $\cdot 119$ & آزمون لامبداى ويلكز \\
\hline $1 / \cdots$ & $\cdot 1 \Lambda$ & $\cdot 1 \cdots 1$ & $\mid f / F$ & rI & f & $r / \cdot r$ & آزمون اثر هتلينتَ \\
\hline $1 / \cdots$ & $\cdot 11$ & $\cdot 1 \cdots 1$ & $\mid f / F$. & rl & r & $f / \cdot r$ & آزمون بزركترين ريشه روى \\
\hline
\end{tabular}

در برقرارى تعادل زيستى-روانى، در شرايط خطرناك مىباشد [بr].

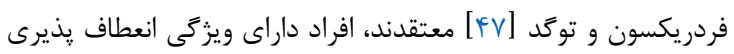

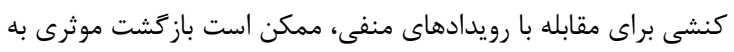

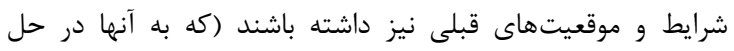
جالشهاى فعلى كمك مى كند) در حالى كه افراد فاقد اين ويزّكى، كويا

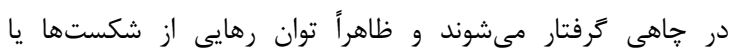

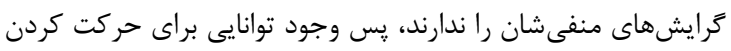

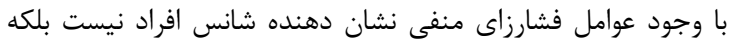

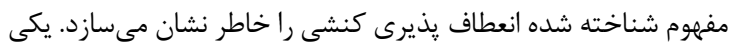

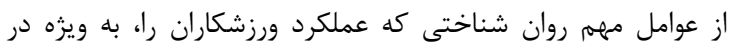

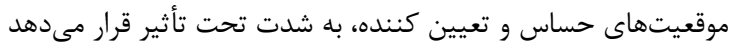

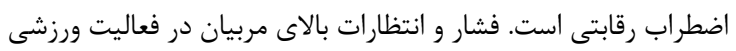

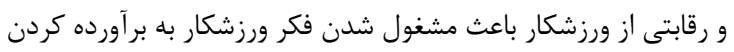

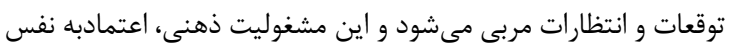

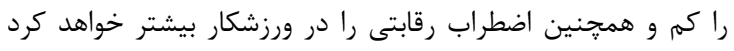

[i $\uparrow \wedge$ ]
در تبيين اين يافته مىتوان كفت كه ورزش مىتواند يردازش هيجانى

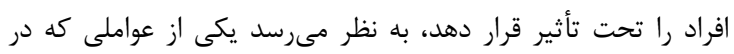

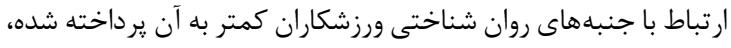
تنظيم شناختى هيجان مى باشد. توضيح اين كه، تنظيمى هيجان وجنه

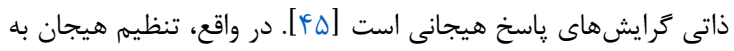

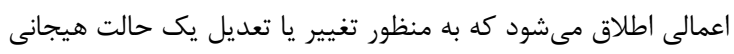

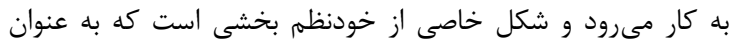

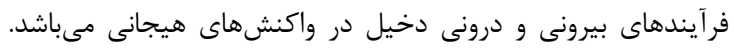

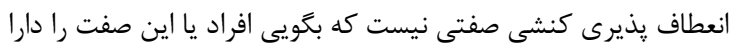

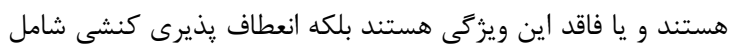

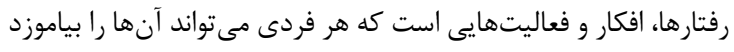

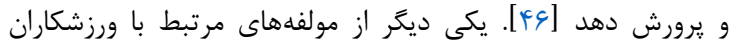

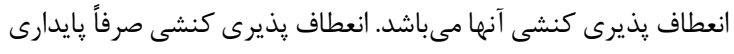

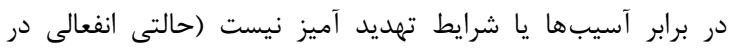

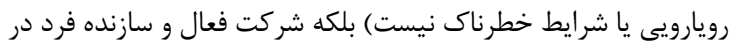

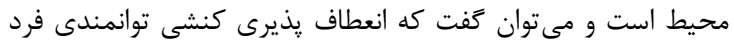


آكَاهى مى تواند به افراد در بهبود جنبه هاى مختلف زندگى به خصوص در بهبود تفكر خلاق و هيجانات تحصيلى دانش آموزان نقش مفئ مفيد
در جهت بهبود تنظيم شناختى هيجان، انعطاف يذيرى كنشى و

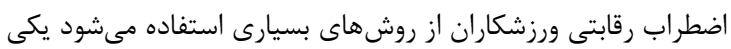

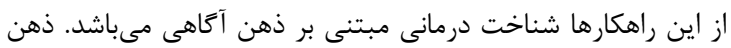

جدول ₹: نتايج تحليل كواريانس يك راهه در متن مانكوا بر روى بسى آزمون تروههاى آزمايش و كواه با كنترل بيشآزمون

\begin{tabular}{|c|c|c|c|c|c|c|c|}
\hline توان آمارى & مجذور اتا & سطح معنى دارى & $\mathbf{F}$ & ميانَين مجذورات & درجه آزادى & مجموع مجذورات & منبع تغييرات \\
\hline \multirow[t]{4}{*}{$1 / \cdots$} & & $\cdot 1 \cdots \cdot 1$ & & & & & تنظيم شناختى هيجان مثبت \\
\hline & $\cdot / A V$ & & $1 \Delta 9 / V V$ & GYN/TA & 1 & GTA/TA & يِيش آزمون \\
\hline & $.19 \Lambda$ & & $\Lambda T / 1 T$ & $r T r / \cdot r$ & 1 & $r r r / \cdot r$ & كروه \\
\hline & & & & r/q r & rV & IFA/QT & خطا \\
\hline \multirow[t]{4}{*}{$1 / \cdot$} & & $\cdot / \cdots 1$ & & & & & تنظيم شناختى هيجان منفى \\
\hline & $\cdot|V|$ & & $9 r / 11$ & $\uparrow q \Delta / V \varphi$ & 1 & $4 q \Delta / V q$ & بيش آزمون \\
\hline & $\cdot / 4 \Lambda$ & & TH/VT & $1 \wedge 9 / V \Delta$ & 1 & I $19 / V Q$ & 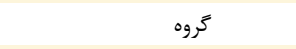 \\
\hline & & & & $\Delta / T V$ & tr & $191 / 91$ & 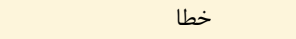 \\
\hline \multirow[t]{4}{*}{$1 / \cdots$} & & $\cdot / \cdots 1$ & & & & & انعطاف يذيرى كنشى \\
\hline & $\cdot / 19$ & & TTY/VF & VQTERT & 1 & TOTY/VQ & ي ״يش آزمون \\
\hline & $\cdot / 4 \Lambda$ & & $r F / \mathscr{G V}$ & rVq/ar & 1 & rVq/Dr & 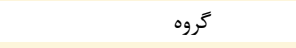 \\
\hline & & & 1.194 & $1 . / 94$ & rV & $4 \cdot 4 / 99$ & خطا \\
\hline \multirow[t]{4}{*}{$1 / \cdot$} & & $\cdot \mid \cdots 1$ & & & & & اضطراب رقابتى \\
\hline & $\cdot|\Lambda|$ & & $19 \cdot 1 \cdot 9$ & $\Delta \cdot \cdot \mid \Delta \Lambda$ & 1 & $\Delta \cdot \cdot \mid \Delta \Lambda$ & 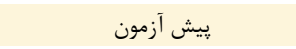 \\
\hline & $\cdot 199$ & & $V M / T F$ & $r 99 / \cdot \Delta$ & 1 & $r q 9 / \cdot \Delta$ & تروه \\
\hline & & & & $r / T$ & tr & $\|\Delta / V\|$ & خطا \\
\hline
\end{tabular}

جدول ه: نتايج تحليل كواريانس جند متغيرى ميانكين نمرات بيكيرى تنظيم شناختى هيجان، انعطاف يذيرى كنشى و اضطراب رقابتى

\begin{tabular}{|c|c|c|c|c|c|c|c|}
\hline توان آمارى & مجذور اتا & سطح معنى دارى P & $\mathbf{F}$ & ميانغين مجذورات & درجه آزادى & مجموع مجذورات & منبع تغييرات \\
\hline \multirow[t]{4}{*}{$1 / \cdot \cdot$} & & $.1 \cdot \cdots 1$ & & & & & تنظيم شناختى هيجان مثبت \\
\hline & $\cdot / \Lambda$ & & $\mid \& q / 94$ & $94 \cdot / 9 T$ & 1 & $94 \cdot / 9 r$ & يِيش آزمون \\
\hline & . lat & & $9 Y / 99$ & TEN/Fi & 1 & $T \& \Lambda / F I$ & 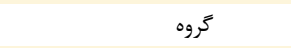 \\
\hline & & & & $F / T \Lambda$ & TV & $\mid \Delta N / F V$ & 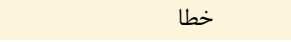 \\
\hline \multirow[t]{4}{*}{$1 / \cdot \cdot$} & & $\cdot|\cdots|$ & & & & & تنظيم شناختى هيجان منفى \\
\hline & $\cdot / \Delta V$ & & $4 q / \cdot 9$ & $r \cdot r / v q$ & 1 & $r \cdot r / v q$ & ي يِش آزمون \\
\hline & $\cdot / \Delta F$ & & $F r / A l$ & $r v \cdot / 4 t$ & 1 & $r v \cdot / q r$ & 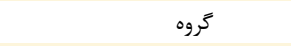 \\
\hline & & & & $9 / 1 \mathrm{~V}$ & rV & TYN/TS & 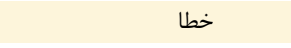 \\
\hline \multirow[t]{4}{*}{$1 / \cdot$} & & $\cdot 1 \cdot \cdots 1$ & & & & & انعطاف يذيرى كنشى \\
\hline & $\cdot / \Lambda \Lambda$ & & rqT/Qf & $r \Delta \Delta N / 9 \Delta$ & 1 & $r \Delta \Delta \Lambda / 9 \Delta$ & ي يَش آزمون \\
\hline & . & & 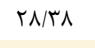 & TFT/G. & 1 & Tसт/G. & كروه - اه \\
\hline & & & & $|r /|$ & rV & FFV/qF & خطا \\
\hline \multirow[t]{4}{*}{$1 / \cdot \cdot$} & & $\cdot \mid \cdot \cdot 1$ & & & & & اضطراب رقابتى \\
\hline & .199 & & VI/91 & $r \wedge r / \cdot r$ & 1 & $r \Lambda r / \cdot r$ & ي بيش آزمون \\
\hline & $\cdot / \Delta T$ & & FI/AT & $r r \cdot / 99$ & 1 & $r T \cdot / 99$ & 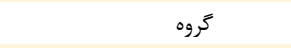 \\
\hline & & & & $\Delta / T T$ & TV & $199 / 1 \mathrm{~V}$ & خطا \\
\hline
\end{tabular}

جدول 9: نتايج تحليل كواريانس يكى راهه در متن مانكوا نمرات ييخيرى تنظيم شناختى هيجان، (مثبت ومنفى) انعطاف يذيرى كنشى و اضطراب رقابتى تروههها

\begin{tabular}{|c|c|c|c|c|c|c|c|}
\hline توان آمارى & مجذور اتا & سطح معنى دارى (P) & $\mathbf{F}$ & خط DF & DF فرضيه DF & مقدار & ن آزمون \\
\hline $1 / \cdot$ & . /VT & $\cdot|\cdots|$ & $9 / r$ & rI & r & $\cdot / N T$ & آزمون اثر ِي يلايى \\
\hline $1 / \cdot$ & $\cdot / V T$ & $\cdot|\cdots|$ & 9/זr & ri & r & $\cdot / r V$ & آزمون لامبداى ويلكز \\
\hline $1 / \cdot$ & ./VT & $\cdot \mid \cdots \cdot$ & 9/rr & rl & r & $r / 9 \mid$ & آزمون اثر هتلينََ \\
\hline $1 / \cdot \cdot$ & $\cdot / \mathrm{r}$ & $\cdot 1 \cdot \cdots 1$ & $9 / \pi r$ & rI & r & $r / 9 \mid$ & آزمون بزركترين ريشه روى \\
\hline
\end{tabular}

طريق آكاهى لحظه به لحظه از افكار، احساسات و حالتهاى جسمانى

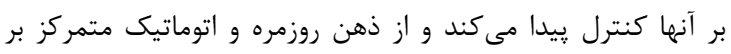

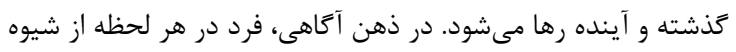

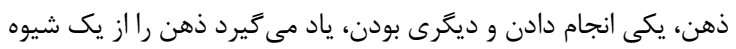

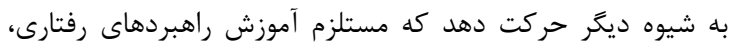

ذهن آكاهى مىتواند آكاهى عميقتر نسبت به احساسات و افكار دشوار

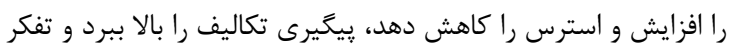

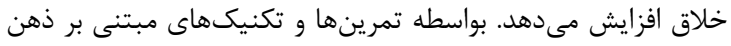

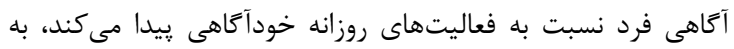

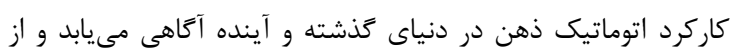




$$
\begin{aligned}
& \text { تنظيم هيجان، انعطاف يذيرى كنشى و اضطراب رقابتى براى }
\end{aligned}
$$

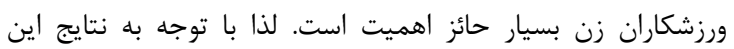

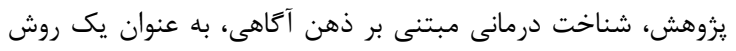

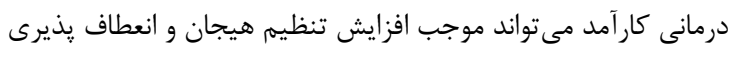

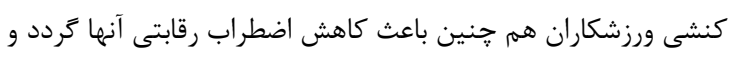

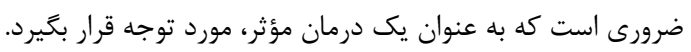

$$
\begin{aligned}
& \text { سياسگزارى }
\end{aligned}
$$

بدين وسيله از تمام مراكز و آزمودنى هاى شركت كننده در يزوهش

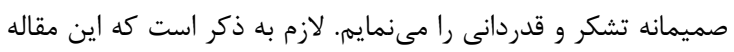

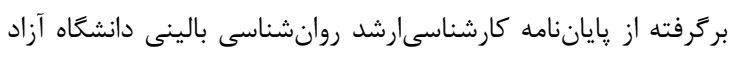
اسلامى واحد اهواز مىباشد. تصند تضاد منافع

هيج كونه تضاد منافعى براى نويسند

\section{References}

1. Abolghasemi A, Kyimarzi A, Aryapouran S, Dargad F. Investigating the relationship between role ambiguity, role conflict and competitive anxiety with sport performance and academic achievement of athlete students. QJ Res Plan Higher Educ. 2006;12(2):39-53.

2. Rostami C, Jahangirlo A, Sohrabi A, Ahmadian H. The Role of Cognitive Flexibility and Mindfulness in Student Neglect Prognosis. J Kurdistan Univ Med Sci. 2016:5061.

3. Kaviani H, Hatami N, Shafiabadi A. The Effect of Cognitive Therapy Based on Mindfulness on the Quality of Life of Depressed People (Non-Clinical). Orig Res Pap Cogn Sci New Years. 2008(4):39-48.

4. Segal ZV, Teasdale JD, Williams JM. Mindfulness Based cognitive therapy for depression. New York: The Guilford Press; 2002.

5. Baer RA. Mindfulness training as a clinical intervention: A conceptual and empirical review. Clin Psychol Sci Pract. 2003;10(2):125-43. doi: 10.1093/clipsy.bpg015

6. Teasdale JD, Segal ZV, Williams JM, Ridgeway VA, Soulsby JM, Lau MA. Prevention of relapse/recurrence in major depression by mindfulness-based cognitive therapy. J Consult Clin Psychol. 2000;68(4):615-23. pmid: 10965637

7. Lykins EL, Baer RA. Psychological functioning in a sample of long-term practitioners of mindfulness meditation. J Cogn Psychother. 2009;23(3):226-41. doi: 10.1891/0889-8391.23.3.226

8. Scherer K. What are emotions? And how can they be measured? Soc Sci Inf. 2005;44(4):695-729.

9. Malouf ET, Youman K, Stuewig J, Witt EA, Tangney JP. A Pilot RCT of a Values-Based Mindfulness Group Intervention with Jail Inmates: Evidence for Reduction in Post-Release Risk Behavior. Mindfulness (N Y). 2017;8(3):603-14. doi: 10.1007/s12671-016-0636-3 pmid: 29242720

10. Thayer JF, Lane RD. A model of neurovisceral integration in emotion regulation and dysregulation. J Affect Disord. 2000;61(3):201-16. pmid: 11163422

11. Mennin DS, Heimberg RG, Turk CL, Fresco DM. Preliminary evidence for an emotion dysregulation model of generalized anxiety disorder. Behav Res Ther. 2005;43(10):1281-310. doi: 10.1016/j.brat.2004.08.008 pmid: 16086981

$$
\begin{aligned}
& \text { شناختى و فراشناختى ويزه براى متمركز كردن فرآيند توجه است [ب]. }
\end{aligned}
$$

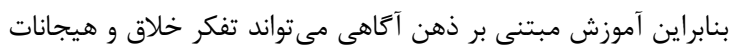

$$
\begin{aligned}
& \text { تحصيلى دانش آموزان را افزايش دهد. }
\end{aligned}
$$

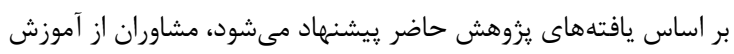

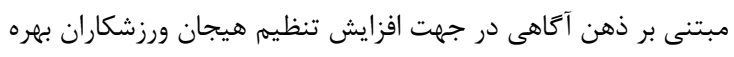

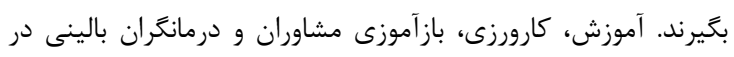

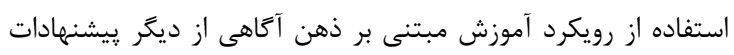

$$
\begin{aligned}
& \text { كاربردى اين يزوهش است. } \\
& \text { نتيجه }
\end{aligned}
$$

با توجه به جمعيت حاضر كشور كه حدود نيمى از آنها را بانوان در

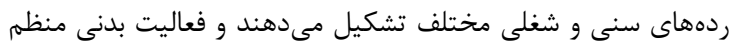

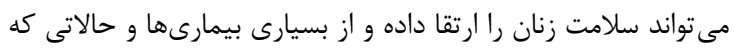

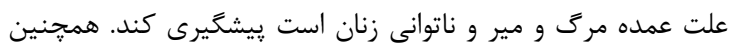

12. Salters-Pedneault K, Roemer L, Tull MT, Rucker L, Mennin DS. Evidence of broad deficits in emotion regulation associated with chronic worry and generalized anxiety disorder. Cogn Ther Res. 2006;30(4):469-80.

13. Tull MT, Barrett HM, McMillan ES, Roemer L. A preliminary investigation of the relationship between emotion regulation difficulties and posttraumatic stress symptoms. Behav Ther. 2007;38(3):303-13. doi: 10.1016/j.beth.2006.10.001 pmid: 17697854

14. Tull MT, Rodman SA, Roemer L. An examination of the fear of bodily sensations and body hypervigilance as predictors of emotion regulation difficulties among individuals with a recent history of uncued panic attacks. J Anxiety Disord. 2008;22(4):750-60. doi: 10.1016/j.janxdis.2007.08.001 pmid: 17851033

15. Kashdan TB, Zvolensky MJ, McLeish AC. Anxiety sensitivity and affect regulatory strategies: individual and interactive risk factors for anxiety-related symptoms. J Anxiety Disord. 2008;22(3):429-40. doi: 10.1016/j.janxdis.2007.03.011 pmid: 17449221

16. McCarthy CJ, Lambert RG, Moller NP. Preventive resources and emotion regulation expectancies as mediators between attachment and college students' stress outcomes. Int J Stress Manage. 2006;13(1):1.

17. Leahy RL. Emotion Regulation in Psychotherapy. New York: Guilford Press; 2012.

18. Barrett LF, Gross J, Christensen TC, Benvenuto M. Knowing what you're feeling and knowing what to do about it: Mapping the relation between emotion differentiation and emotion regulation. Cogn Emot 2001;15(6):713-24. doi: 10.1080/02699930143000239

19. Yoo SH, Matsumoto D, LeRoux JA. The influence of emotion recognition and emotion regulation on intercultural adjustment. Int $\mathrm{J}$ Intercult Relat. 2006;30(3):345-63. doi: 10.1016/j.ijintrel.2005.08.006

20. Garnefski N, Kraaij V. Cognitive emotion regulation questionnaire-development of a short 18-item version (CERQ-short). Pers Individ Differ. 2006;41(6):1045-53. doi: 10.1016/j.paid.2006.04.010

21. Lazarus RS. Coping theory and research: past, present, and future. Psychosom Med. 1993;55(3):234-47. pmid: 8346332 
22. Martin RC, Dahlen ER. Cognitive emotion regulation in the prediction of depression, anxiety, stress, and anger. Pers Individ Differ. 2005;39(7):1249-60. doi: 10.1016/j.paid.2005.06.004

23. Agaibi CE, Wilson JP. Trauma, PTSD, and resilience: a review of the literature. Trauma Violence Abuse. 2005;6(3):195-216. doi: 10.1177/1524838005277438 pmid: 16237155

24. Wahhabi M, Shahmoradi M, Sheikh S. Comparison of Critical Thinking, Hard Work and Flexibility of the Students of Payame Noor University, Abdanan. J Psychol Educ Sci. 2016; 7:109-22.

25. Hamill SK. Resilience and self-efficacy: The importance of efficacy beliefs and coping mechanisms in resilient adolescents. Colgat Univ J Sci. 2003;35(1):115-46.

26. Siebert A. How to develop resiliency stregths 2007 . Available from: www.resiliencycenter.com.

27. Connor KM, Davidson JR. Development of a new resilience scale: the Connor-Davidson Resilience Scale (CD-RISC). Depress Anxiety. 2003;18(2):76-82. doi: 10.1002/da.10113 pmid: 12964174

28. Speilberger AE, Olewine DA. The effect of an intrauterine suture on pregnancy and deciduomata formation in the rat. Int J Fertil. 1966;11(1):15-9. pmid: 5936281

29. Hanin YL. A Study of Anxiety in Sport, In W.F. Straub (Ed.), Sport Psychology: An Analysis of Athlete Behavior, Ithaca. New York: Movement; 1980.

30. Krane V. A practical application of the anxiety-athletic performance relationship: The Zone of Optimal Functioning hypothesis. Sport Psychol. 1993;7(2):11326. doi: $10.1123 /$ tsp.7.2.113

31. Fletcher D, Hanton S. The relationship between psychological skills usage and competitive anxiety responses. Psychol Sport Exerc. 2001;2(2):89-101.

32. Hanton S, Thomas O, Maynard I. Competitive anxiety responses in the week leading up to competition: the role of intensity, direction and frequency dimensions. Psychol Sport Exerc. 2004;5(2):169-81. doi: 10.1016/S14690292(02)00042-0

33. Kahrizi S, Taghavi M, Ghasemi R, Goodarzi M. The effectiveness of mindfulness-based cognitive therapy (MBCT) on epression, Anxiety and Somatic Symptoms in Asthma patients. Razi J Med Sci. 2017;24(154):27-36.

34. Kord B, Rahbari P. The Prediction of Subjective Wellbeing Based on Meaning of Life and Mindfulness among Cardiovascular Patients. Iran J Pediatr Nurs. 2018;5(6):16-23.

35. Pourmohammadi S, Bagheri F. The Effectiveness of Mindfulness Training on Automatic Cognitive Processing. Psychol Stud. 2015;11(3):141-59.
36. Hartmann M, Kopf S, Kircher C, Faude-Lang V, Djuric Z, Augstein F, et al. Sustained effects of a mindfulness-based stress-reduction intervention in type 2 diabetic patients: design and first results of a randomized controlled trial (the Heidelberger Diabetes and Stress-study). Diabetes Care. 2012;35(5):945-7. doi: 10.2337/dc11-1343 pmid: 22338101

37. Crane R. Cognitive Therapy Based on Presence of Mind. Tehran: University of Social Welfare and Rehabilitation Sciences; 2012.

38. Kabat-Zinn J. Mindfulness-based interventios in content: past, present and future. Clin Psychol Sci Pract. 2010;10(2):144-56.

39. Mohammadi M. Investigating factors affecting resilience in people at risk of drug abuse. Tehran: University of Welfare and Rehabilitation Sciences; 2005.

40. Samani S, Jokar B, Sahragard N. Effects of resilience on mental health and life satisfaction. Iran J Psychiat Clin Psychol. 2007;13(3):290-5.

41. Martens R, Vealey RS, Burton D. Competitive Anxiety in Sport. Champaign, llinois: Human Kinetics; 1990.

42. Garnefski N, Kraaij V, Spinhoven P. Negative life events, cognitive emotion regulation and emotional problems. Pers Individ Differ 2001;30(8):1311-27.

43. Yousefi F. The relationship of cognitive emotion regulation strategies with depression and anxiety in students of special middle schools for talented students in Shiraz. J Except Child. 2007;6(4):871-92.

44. Yousefi F. The causal model of emotional intelligence, cognitive development, emotional regulation cognitive strategies, and general health: Shiraz University; 2004.

45. Ayduk Ö, Kross E. Analyzing negative experiences without ruminating: The role of self-distancing in enabling adaptive self-reflection. Soc Pers Psychol Compass. 2010;4(10):841-54. doi: 10.1111/j.17519004.2010.00301.x

46. Comaz Diaz L, S. L, R. M, K.W. S, G t. The Road to Resilience. Washington: DC. American psychology Association; 2011.

47. Fredrickson BL, Tugade MM, Waugh CE, Larkin GR. What Good Are Positive Emotions In Crises? Personality processes and individual difference. J Pers Soc Psychol. 2004;84(2):365-76.

48. Beyrami M, Movahedi Y, Kazemi Rezaei S, Ismaili S. The Effect of Cognitive-Based Mindfulness-Based Therapy on Reducing Pathological Concerns and Anxiety Symptoms in Patients with Generalized Anxiety Disorder. J Rehabil Res Nurs. 2015;2(1):79-90. 